\title{
Fracture initiation from initial spherical flaws in incompressible propellant materials
}

\author{
Panayiotis A. Kakavas ${ }^{1}$, Alexander V. Perig ${ }^{2}$
}

\author{
${ }^{1}$ Technological Educational Institute of Patras, 1 M. Alexandrou str, 26443,Patras, Greece (Hellas) \\ e-mail: kakavas@teipat.gr \\ ${ }^{2}$ Donbass State Engineering Academy, Shkadinova 72, Donetsk Region, Kramatorsk 84313, Ukraine \\ e-mail: olexander.perig@gmail.com
}

\section{ABSTRACT}

The scope of the present article is the study of the fracture initiation from initial spherical flaws in incompressible propellant materials. Using a constitutive law, derived from the neo-Hookean strain energy function, one may deduce the relation between the radial pressure applied at infinity and the corresponding extension ratio, at the surface of the cavity. An equation which defines the critical extension ratio for a given value of a parameter, $\kappa$, was derived and the corresponding critical load, pc, is related to the critical cavity extension ratio. Plots for the extension ratio and normalized pressure were provided solving the appropriate equation derived from the analysis.

The failure of the cavity is associated with the incremental energy transfer at a constant critical stress between the applied work, internal strain energy and surface energy. The influence of the surface energy on the radial deformation of an initially intact sphere is presented and it turns out that a characteristic cavitation phenomenon occurs instead of the bifurcation. A mathematical formula is derived for the pressure, per unit current area, which is valid for a general form of incompressible elastic strain energy function. Using the derived formula one may deduce the relation between the radial pressure and the corresponding extension ratio at the surface of the cavity. In an incompressible material of given modulus and surface energy it is proved that a critical field stress exists at which a small internal cavity may grow in size where the significant quantities are the radial and tangential strains.

Keywords: Fracture, propellant materials, incompressible solids, rubbers, poker-chip specimen.

\section{List of symbols}

$\begin{array}{ll}a_{0} & \text { Initial radius of a spherical cavity } \\ b_{0} & \text { Initial radius of a spherical medium } \\ a_{1} & \text { Final radius of a spherical cavity } \\ b_{1} & \text { Final radius of a spherical medium } \\ p & \text { Uniform hydrostatic tensile stress } \\ p_{c} & \text { Critical pressure } \\ \bar{p} & \text { Normalized pressure } \\ \varphi & \text { Surface energy per unit current area } \\ \lambda & \text { Principal stretch ratio } \\ \lambda_{c} & \text { Critical stretch ratio } \\ I_{c} & \text { Critical radius } \\ \bar{W} & \text { Strain energy function } \\ \mu & \text { Shear modulus of the material } \\ U_{1} & \text { Potential energy } \\ U_{2} & \text { Internal strain energy in the material } \\ E & \text { Tensile modulus of the material at small strains } \\ I_{1} & \text { First strain invariant }\end{array}$




$\begin{array}{ll}\lambda_{i}(i=1,2,3) & \text { Local principal extension ratios } \\ U_{3} & \text { Surface energy of the cavity per unit area } \\ \kappa & \text { Parameter } \\ \varepsilon & \text { Infinitesimal strains } \\ \lambda_{r}, \lambda_{\theta} & \text { Radial and tangential strains } \\ \sigma_{r}, \sigma_{\theta} & \text { Radial and tangential stresses }\end{array}$

\section{INTRODUCTION}

The microstructural damage and fracture processes associated with the fracture-toughness behavior of a polymer-matrix composite solid rocket-propellant material have been studied [1]. Specifically, nonlinearelastic fracture-mechanics tests were performed, as a function of displacement rate and temperature on center-cracked sheet test samples to determine fracture toughness in the form of J-integral resistance curves and viscoelastic resistance curves for the inert propellant $\mathrm{H}-24$; in addition, in situ video imaging was employed to characterize the deformation and interaction between microstructural features and the crack-path morphology. Experiments have been performed on poker chip type of sample, using the acoustic emission technique, to evaluate the cavitation process in rubber materials [2]. Theoretical studies on the cavitation process in poker chip rubber samples have also been investigated [3] . Values of the stress-intensity factor, $\mathrm{K}_{\mathrm{c}}$, at the onset of crack growth have been measured for a nitrocellulose/nitroglycerine propellant [4]. The application of quasi-static viscoelastic fracture mechanics to composite solid propellant and composite modified double-base propellant has been discussed [5]. Both types are used in modern case-bonded solid rockets. Generally speaking, these propellants consist of a soft matrix which is highly filled with hard particles of varying size. Although solid propellants are nonlinear viscoelastic materials, linear elastic and linear viscoelastic fracture mechanics have met with some success in characterizing fracture behavior. A mechanical model has been developed for the behavior of propellant-like materials under high confining pressure and strain rate [6]. The local behavior near the crack tip and the crack growth behavior in a composite solid propellant containing hard particles in a rubbery matrix, under various loading conditions have been investigated [7].

The effect of pre-damage on the high strain-rate behavior, ignition sensitivity, and impact ignition mechanism of a HTPB/AP rocket propellant has been assessed, using a modified Hopkinson Bar [8]. The pre-damage has characterized using a failure criterion based on a linear cumulative damage model. By means of SEM and micro-dynamic tensile instrument, the fracture cross section microscopic pattern of HTPB propellants has been observed and the fracture behavior of midget tensile samples at strain state has been analyzed [9]. Propellant samples have been recovered from interrupted-burning experiments. Different modes of crack propagation and/or branching were observed. Depending upon the rate of pressurization, these modes are: unidirectional crack propagation; single-crack propagation accompanied by branching from various locations; and multiple branching in various directions from the initial crack-tip region. The crackpropagation problem has analyzed through the use of basic physical principles, experimental results, and Schapery's theory of crack propagation [10]. On the basis of qualitative observation of the effect of different content of bonding agents on the fracture propagation process through the in-situ SEM observations, effect of the bonding agents amount on mechanical properties of NEPE solid propellant have been quantitatively studied through image processing and differential box counting calculating [11].

Using rigorous homogenization theory for composite materials, a general 3-D nonlinear macroscopic constitutive law that models microstructural damage evolution upon straining through continuous void formation and growth has proposed [12]. This law addresses the viscous deformation rate within the framework of additive decomposition of the deformation rate and the concept of back stress is used to improve the model performance in stress relaxation.

Since the conditions under which fracture is initiated are so important in engineering applications, it is necessary to understand them thoroughly. Even if one is mainly concerned with the mechanics of crack propagation the initiation phase or triggering phenomenon is still an important consideration. Since the failures, that have been observed [2] emerge as sudden, catastrophic bursts in which a spherical void appears, one would suspect the existence of initial "residual" voids which grow under applied load until they reach a critical void size at which this burst occurs. We shall therefore postulate the presence of an initial void and idealize it as a spherical cavity of initial radius $\mathrm{a}_{0}$ embedded in the center of a spherical medium of initial radius $b_{0}$ as shown in the sketch below. Here we analyze an unstable expansion of a cavity of initially finite 
size based on the energy balance, while the paper by Biwa [16] was done for cavity growth from zero radius.

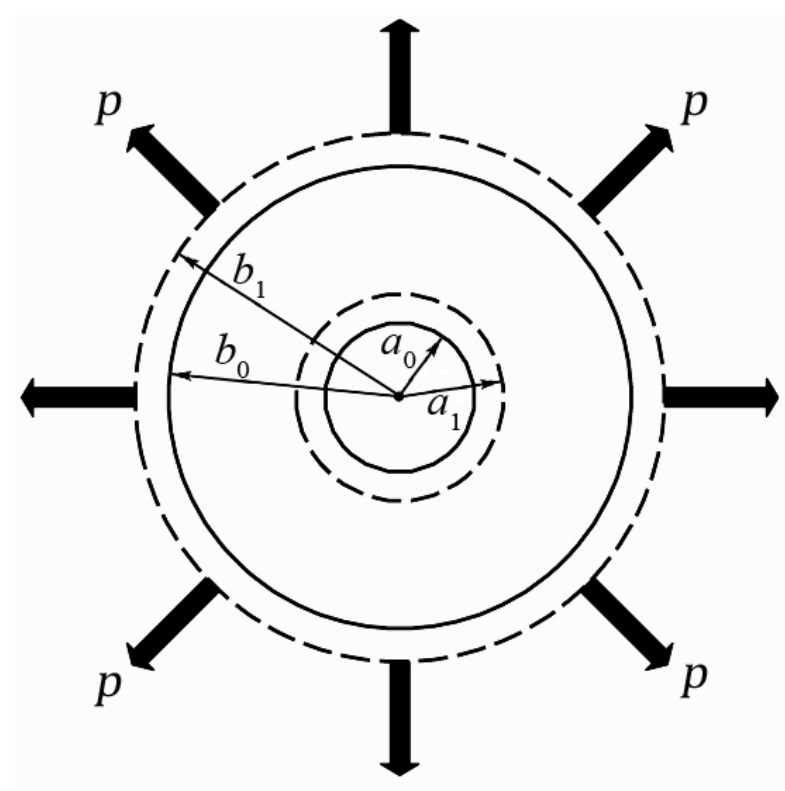

Figure 1: Spherical model under tri-axial stress.

The outside of the medium will be assumed to be subject to a uniform hydrostatic tensile stress, p. Since these cavities will be considered to be small in comparison to the dimensions of the specimen, we will eventually approximate the problem as a spherical cavity in an infinite medium. This will be done in the subsequent analysis by making the ratio of the radii $\left(b_{0} / a_{0}\right)$ be very large compared to unity. In this limit the surface stress p, acting at infinity will be assumed to be the local field stress in the poker-chip specimen. The above assumptions also imply that the voids are sufficiently small and isolated so as to have little interaction with each other. This assumption is therefore similar to the dominant: flaw concept often invoked in brittle fracture.

When an external normal traction is applied, the internal cavity size will increase in such a way that the energy balance between the applied work and the internally stored strain energy will be preserved. On the other hand, one can inquire for conditions under which it is possible for this energy balance to be achieved in an alternate way. In particular, one can visualize increased cavity area arising from the creation, at fracture, of additional surface, a phenomenon which requires an incremental increase in surface energy. In the ensuing analysis, failure of the cavity will be associated with incremental energy transfers at some constant critical stress $p_{c}$ between applied work, internal strain energy, and surface energy. Considerations similar to those used by Griffith [13] will be employed, but in addition finite strains will be permitted because of our desired application to rubbery incompressible media. At this point we should mention the contribution of Professors Ball [14] and Horgan et al [15] and Biwa [16] in the field of cavitation in nonlinear elastic materials. Below we present some of the analysis of this problem, proposed by Biwa, for comparison with our approach.

\section{BIWA'S APPROACH OF SOLVING THE PROBLEM OF CAVITATION IN FINITE ELASTICITY WITH SURFACE EFFECTS [16]}

The analysis proposed by Biwa [16] examines the effect of surface energy on cavitation formation at finite spherically symmetric deformation of isotropic hyperelastic solids. In order to clarify the implication of the stretch dependence on the surface energy, the formulation was first laid down for expansion of a hollow hyperelastic sphere. The influence of the surface energy on the radial deformation of an initially intact sphere was examined where it turns out that a characteristic cavitation phenomenon occurs instead of the bifurcation. A jump from an intact equilibrium to cavitated equilibrium is expected at a certain loading level in order for the sphere to have less potential energy. According to Biwa's analysis the limiting behavior was considered where the initial radius of the sphere vanishes which implies an initially intact incompressible sphere. The response curve for an initially intact incompressible hyperelastic sphere is given by 


$$
p=\lambda^{2} \int_{\lambda}^{\infty} \frac{d \bar{W}}{\frac{d t}{t^{3}-1}} d t+2 \frac{\varphi_{\infty}}{a_{1}}
$$

where $\varphi$ is associated with the surface energy per unit current (deformed) area. It is allow ${ }^{\wedge \lambda}$ to depend on the stretch ratio of the inner surface, namely, $\varphi=\varphi\left(\lambda_{c}\right)$ where the cavity wall stretch $\lambda_{c}$ is given by $\lambda_{c}=a_{1} / a_{0}$. No surface energy is considered to be associated with the outer surface of the sphere. The hollow sphere is associated with certain amount of surface energy even in its initial configuration, which is then not a stress free state as usually understond implicitly in finite elasticity. For the special case of a neo-Hookean material where $\bar{W}$ has the form, $\bar{W}=(\mu / 2)\left(\sum_{\mathrm{i}=1}{ }^{3}\left(\lambda_{\mathrm{i}}{ }^{2}-3\right)\right)$, where $\mu_{\text {defines the shear modulus of the }}$ material, which is approximately equal to one third of the Young's modulus, equation (1) transforms to

$$
\frac{p}{\mu}=2 \lambda+\frac{1}{2 \lambda^{2}}+\frac{\varphi_{\infty}}{b_{0} \mu} \frac{2 \lambda^{2}}{\left(\lambda^{3}-1\right)^{1 / 3}}
$$

Therefore the response depends only on the ratio of the asymptotic value $\varphi_{\infty}$ to $b_{0} \mu$ irrespective of the functional form of $\varphi=\varphi\left(\lambda_{c}\right)$.The ratio $S=\varphi_{\infty} / b_{0} \mu$ represents the normalized surface energies. The parameter $\varphi_{\infty}$ is assumed as a positive finite constant. For more details one can refer to ref. [16].

A plot of the surface traction stretch relation for an initially intact incompressible neo-Hookean sphere, for various values of $S$ is shown in Fig. 2 (see ref. [16]).

\section{DETERMINATION OF THE CRITICAL PRESSURE}

To examine this critical condition a small change $\delta a_{0}$ in the original radius $a_{0}$ is assumed, and the failure condition is specified by the following energy balance equation [17]

$$
\delta(\text { external work })=\delta(\text { surface energy })+\delta(\text { strain energy })
$$

where in the above equation the Greek symbol $\delta$ is to be interpreted as the variation with respect to $a_{0}$ under condition of constant applied stress. The deformed internal and external radii are respectively $a$ and $b$, corresponding to two stretch ratios $\lambda_{a}$ and $\lambda_{b}$ defined as follows

$$
\lambda_{a}=\frac{a}{a_{0}} ; \lambda_{b}=\frac{b}{b_{0}}
$$

In the present analysis it will be assumed that the medium in question is incompressible and therefore the volume will be conserved during elastic deformation. This relates $\lambda_{b}$ to $\lambda_{a}$ by the following relation

$$
\lambda_{b}^{3}=1+\left(\frac{a_{0}}{b_{0}}\right)^{3}\left(\lambda_{a}^{3}-1\right)
$$

It may be noted that for the configuration with which we are concerned, one has $\left(a_{0} / b_{0}\right)<<1$ and also $\lambda_{a}\left(a_{0} / b_{0}\right)<<1$, so that we have, approximately

$$
\lambda_{b}=1+\frac{1}{3}\left(\frac{a_{0}}{b_{0}}\right)^{3}+\left(\lambda_{a}^{3}-1\right)+\ldots \ldots
$$


and

$$
\lambda_{b}^{2}=1+\frac{2}{3}\left(\frac{a_{0}}{b_{0}}\right)^{3}+\left(\lambda_{a}^{3}-1\right)+\ldots \ldots
$$

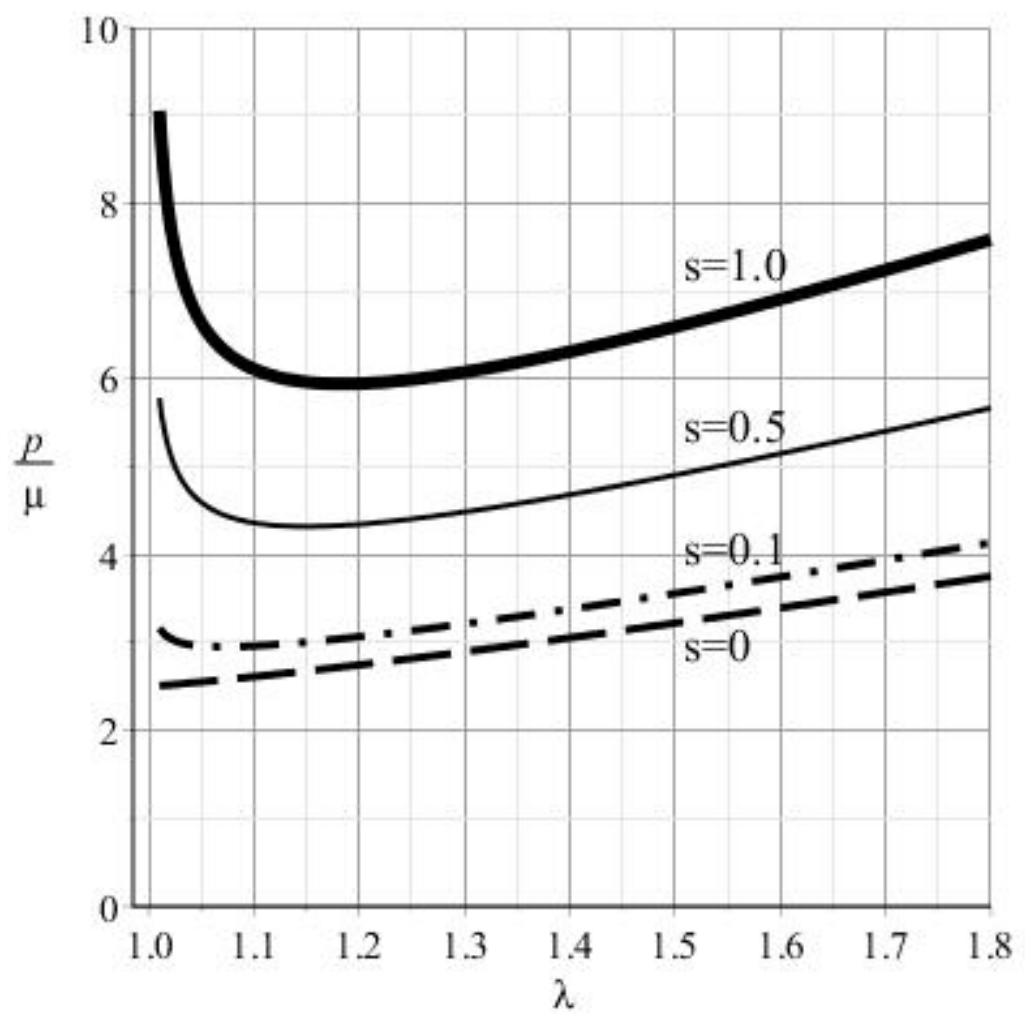

Figure 2: Plot of $p / \mu$ as a function of $\lambda$ for different values of the normalized surface energy $S=\varphi_{\infty} / \mathrm{b}_{0} \mu$.

The potential energy $U_{1}$ of the external load $\mathrm{p}$, is equal to the total load acting on the external boundary of the medium times the displacement of that boundary. Therefore

$$
U_{1}=\int_{b_{0}}^{b} 4 \pi b^{2} p d r=\frac{4}{3} \pi p\left(b^{3}-b_{0}^{3}\right)
$$

or using eqns (5) and (7)

$$
U_{1}=\frac{4 \pi}{3} a_{0}^{3} p\left(\lambda_{a}^{3}-1\right)
$$

The internal strain energy in the material $U_{2}$, is equal to total work done by the external surface force $p$, during the deformation, assuming no new fracture surface is created. To calculate this energy, the relation between the external load and deformation is required, and this depends on the constitutive laws of the material. For the purpose of the present analysis, it is assumed that the material is Neo-Hookean, so that it can be characterized by a strain energy function of the form [18] 


$$
W=\frac{E}{6}\left(I_{1}-3\right)
$$

where in this expression $E$, is the tensile modulus as measured at small strains and $I_{1}$ is the first strain invariant

$$
I_{1}=\sum_{i=1}^{3} \lambda_{1}^{i}
$$

written for this spherical configuration in terms of the local principal extension ratios

$$
\lambda_{1}=\lambda_{r} ; \lambda_{2}=\lambda_{\theta} ; \lambda_{3}=\lambda_{\phi}
$$

where the conditions of symmetry require $\lambda_{\theta}=\lambda_{\phi}$. The Neo-Hookean material assumption implies incompressibility, i. e.

$$
\prod_{i=1}^{3} \lambda_{i}=1 \text { or } \lambda_{r} \lambda_{\theta}^{2}=1
$$

so that, upon substitution into (10), yields

$$
W=\frac{E}{6}\left(2 \lambda_{\theta}^{2}+\lambda_{\theta}^{-4}-3\right)
$$

The spherically symmetric deformation of a shell of incompressible material can be written as

$$
r=R f(R)
$$

where for unconstrained materials the function $f(R)$ is given by [see ref [1] ]]

$$
f(R)=\left(1+\frac{a^{3}-a_{0}^{3}}{R^{3}}\right)^{1 / 3}=\lambda_{\theta}
$$

The radial equation of equilibrium is defines by [타 $]$

$$
\frac{d t_{r}^{(1)}}{d R}+\frac{2}{R}\left(t_{r}^{(1)}-t_{\theta}^{(1)}\right)=0
$$

For isotropic materials and principal axes we have

$$
t_{r}^{(1)}=\frac{\partial W}{\partial \lambda_{r}}-p \lambda_{r}^{-1} ; t_{\theta}^{(1)}=\frac{\partial W}{\partial \lambda_{\theta}}-p \lambda_{\theta}^{-1}
$$


where $\lambda_{r}$ and $\lambda_{\theta}$ are related via equation (13).

From eqn(16) we obtain that

$$
R \frac{d \lambda}{d R}=-\left(\lambda_{\theta \theta}-\lambda^{-2}\right)
$$

Using eqn (19) eqn (17) becomes

$$
\frac{d t_{r}^{(1)}}{d \lambda}=-\frac{2}{\left(\lambda_{\theta}-\lambda_{\theta}^{-2}\right)}\left(t_{r}^{(1)}-t_{\theta}^{(1)}\right)
$$

Now, we assume that the boundary $R=b_{0}$ is free of traction while $R=a_{0}$ is subjected to a pressure $P$, per unit current area. Hence, the boundary conditions are

$$
t_{r}^{(1)}=-p \lambda_{a}^{2} \text { for } R=a_{0} \text { and } t_{r}^{(1)}=0 \text { for } R=b_{0}
$$

On introduction of the notation

$$
\bar{W}=W\left(\lambda_{\theta}^{-2}, \lambda_{\theta}^{-1}, \lambda_{\theta}\right)
$$

using eqns (18) we obtain

$$
t_{\theta}^{(1)}-\lambda_{\theta}^{-3} t_{r}^{(1)}=\frac{1}{2} \frac{d \bar{W}}{d \lambda_{\theta}}
$$

and integration of eqn (20) with the help of eqn (21) then yields the pressure $P$ equal to

$$
p=\int_{\lambda_{a}}^{\lambda_{b}} \frac{d \bar{W}}{d \lambda_{\theta}} d \lambda_{\theta}
$$

The equation (24) is valid for a general form of incompressible elastic strain energy function. Using this constitutive law (24) one may deduce the relation between the radial pressure applied at infinity and the corresponding extension ratio, at the surface of the cavity, $\lambda_{a}$,

$$
p=\frac{E}{6}\left(5-4 \lambda_{a}^{-1}-\lambda_{a}^{-4}\right)
$$

This for small strains eqn (25) yields the appropriate limit value

$$
p=\frac{4 E}{3}\left(\lambda_{a}-1\right)
$$

Therefore the strain energy stored in the medium is

$$
U_{2}=\int_{1}^{\lambda_{b}} 4 \pi p b_{0}^{3} \lambda_{b}^{2} d \lambda_{b}
$$


Assuming that $\left(a_{0} / b_{0}\right)<<1$ and using relations (6), (7) and (25) then (27) can be written as

$$
U_{2}=\int_{1}^{\lambda_{b}} 4 \pi b_{0}^{3} \frac{E}{6}\left(5-4 \lambda_{a}^{-1}-\lambda_{a}^{-4}\right) \lambda_{a}^{2} d \lambda_{a}
$$

By carrying out the integration yields that $U_{2}$ is equal to

$$
U_{2}=\frac{2 \pi E a_{0}^{3}}{3}\left(\frac{5}{3} \lambda_{a}^{3}-2 \lambda_{a}^{2}+\lambda_{a}^{-1}-\frac{2}{3}\right)
$$

For the undeformed state $\lambda_{a}=1$ and the above equation yields $U_{2}=0$ as it should be!

The surface energy of the cavity $U_{3}$ is defined by

$$
U_{3}=4 \pi a_{0}^{2} S
$$

where $S$ is the surface energy per unit area. Physically the quantity $S$, which was first introduced for crack problems by Griffith [13] , can be interpreted as the energy required performing the necessary molecular bond breaking in order to produce a unit area of new surface. Using the criterion for the fracture of the cavity,

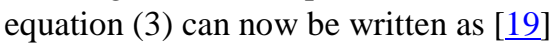

$$
\delta\left(U_{1}\right)=\delta\left(U_{2}\right)-\delta\left(U_{3}\right)
$$

where obviously the fact was used that the small change in work done by external force is equal to the change in its strain energy. Here we should mention that the approach proposed by Biwa [16] is completely different than the one we deal in the present paper.

Substituting into equation (31) from (9), (29) and (30) and recalling that the variation is carried out at constant pressure with respect to the unfractured radius $a_{0}$, it follows that

$$
\delta\left(\frac{4 \pi}{3} a_{0}^{3} p\left(\lambda_{a}^{3}-1\right)\right)=\delta\left(\frac{2 \pi E}{3} a_{0}^{3}\left(\frac{5}{3} \lambda_{a}^{3}-2 \lambda_{a}^{2}+\lambda_{a}^{-1}-\frac{2}{3}\right)\right)-\delta\left(4 \pi a_{0}^{2} S\right)
$$

From equation (25) it can easily be seen that $p$ is a rnonotonically varving function of $\lambda_{a}$ and therefore the above variation under the rondition of constant pressure implies that $\lambda_{a}$ is also constant. Therefore varying eqn (30) with respect to $a_{0}$ gives

$$
a_{0}\left[p a_{0}\left(\lambda_{a}^{3}-1\right)-2 S-\frac{E a_{0}}{2}\left(\frac{5}{3} \lambda_{a}^{3}-2 \lambda_{a}^{2}+\lambda_{a}^{-1}-\frac{2}{3}\right)\right]=0
$$

One can defines the constant $\kappa$ as follows

$$
\kappa=\frac{6 S}{E a_{0}}
$$

The parameter $\kappa$ in our methodology is related to the surface energy for a cavity within a defnrmed material, under the influence of the load, $P$. According to Biwa's [16] approach the surface energy $\varphi_{\infty}$ is related to the intact cavity in the solids. Therefore these two methods approach the problem in a different way. The 
results in Fig. 2, show that the dead load traction $p$, above a certain level, there are multiple equilibrium solutions, namely, the intact configuration and two cavitated configurations. The associated energies of the two types of solutions are compared to each other to determine the energetically favorable equilibrium solution for a given $P$.

And equation (33) can be rewritten as follows

$$
\lambda_{a}^{6}-\left(\frac{3}{2}+\kappa\right) \lambda_{a}^{4}+\frac{1}{2}=0
$$

The above equation defines the critical extension ratio for a given value of the parameter $\kappa$. The corresponding critical load $p_{c}$ is related to the critical cavity extension ratio, $\lambda_{a c}$ by eqn (25).

\section{CRITICAL FLAW SIZES}

Solutions to equations (35) will now be investigated. In general, this equation can be solved analytically [20] but here it is proferred to solve the equation numerically. Figure 3, presents the results for the critical extension ratio $\lambda_{a c}$ and critical external load $6 \mathrm{p}_{\mathrm{c}} / 5 E$ against the parameter $\kappa$ on a semi-log scale. For the two extreme ranges of $\kappa$, that is for $\kappa<<1$ and $\kappa>>1$, approximate analytic expressions which can be obtained for the critical extension ratio and the load.

Case I: In the range $\kappa<<1$ i.e. larger cavities, where the surface tension is relativoly small or the voids reasonably large, it may be verified that equation (35) can be closely satisfied by $\lambda_{a c}$. To determine this root, one can write

$$
\lambda_{a c}=1+\varepsilon
$$

where $\varepsilon<<1$. Upon substituting (34) into (33) and neglecting terms of order $\varepsilon^{3}$ or higher it follows that

$$
\varepsilon=\sqrt{\frac{\kappa}{6}}=\sqrt{\frac{S}{E a_{0}}}
$$

from which

$$
\lambda_{a c}=1+\sqrt{\frac{\kappa}{6}}
$$

The associated value of the critical pressure, $p_{c}$, using eqn (25), is

$$
p_{c}=\frac{4 \pi}{3} \sqrt{\frac{\kappa}{6}}=\frac{4}{3} \sqrt{\frac{S E}{a_{0}}}
$$

which is valid for infinitesimal strains.

One may firct observe that this expression has the familiar form of the Griffith critical stress [13], wherein the radius $a_{0}$ plays the part of the half crack length, $C$, in an infinite sheet. For reference, this latter situation gives

$$
p_{c}=\sqrt{\frac{2 S E}{\pi c}}
$$


so that the numerical factor on the critical pressure differs by only 50 percent, reflecting the curvature and biaxial stress field at the flaw. Note further that it is possible to obtain an excellent estimate of the importance of small vs. large strain theory by examining the departure of the actual extension ratio from the infinitesimal estimate.

Case II: At the other end of the scale, $\kappa>>1$, smaller cavities, where the surface tension is large compared to the modulus or there exist very small flaws, it is also possible to obtain the approximate root of (35) as

$$
\lambda_{a c}=\sqrt{\kappa}=\sqrt{\frac{6 S}{E a_{0}}}
$$

and the corresponding expression for the critical load is

$$
\frac{p_{C}}{E}=\frac{5}{6}\left(1-\sqrt{\frac{8 E a_{0}}{75 S}}\right)
$$

The error in equation (42) is 2.5 percent at $\kappa=50$ and it decreases as $\kappa$ increases and remembering that $\kappa=6 S / E \mathrm{~g}$ the above results indicate an interesting point. For a given value of $E / 6 S$ it can be seen from equation (41) that as the initial radius $a_{0}$ decreases, the critical extension ratio $\lambda_{a c}$ increases and it becomes infinite for vanishingly small cavity radius, although at a finite critical applied pressure of $5 E$ / 6 it can be noted that the high stretch ratios obtained for small voids at fracture confirms the need for finite elastic theory for such geometries. In conclusion. it is imnortant to note that the above asymptotic approximations are valid on either end of a 6-decade band, $10^{-2}<<\kappa<<10^{-3}$ as can be readily seen in Figure 3 .

For the intermediate transition region the values of critical applied pressure and associated cavity strain may be obtained from the curve. The SCILAB computer program has been used for this analysis. Equation (35) solved for $\kappa$ and various values of the critical principal stretch $\lambda_{a}$ was assigned, and the corresponding values of $\kappa$ were computed. A plot of $\lambda_{a}$ vs. $\log (\kappa)$ was produced and the result is shown in Figure 3. In the same Figure 3, a plot of the normalized pressure $\bar{p}=6 p_{c} / 5 E$ as a function of $\log (\kappa)$ is plotted too. At large values of $\kappa$, approximately $10^{3}$, the normalized pressure approaches unity.

\section{TRIAXIAL FAILURE ANALYSIS}

It has been shown that in an incompressible material of modulus $E$ and cirface energy $S$ there exists a potential critical field stress $p$ at which a small internal cavity of radius $a_{0}$ may grow in size. Because the relation between the field stress and the stress and strain state at the cavity has also been determined, knowledge of the local conditions at the point of fracture is available to examine the validity of various theories of failure, such as those enumerated in the first section. At the cavity, the significant quantities are the radial and tangential strains [21, 22]

$$
\begin{aligned}
& \text { Circumferential strains: } \lambda=\lambda_{a} \\
& \text { Radial strain: } \lambda_{r}=\lambda_{\theta}^{-2}=\lambda_{a}^{-2} \text { (incompressibility condition) }
\end{aligned}
$$

and using the constitutive law (14), one can find the expression for the circumferential stress $\sigma_{\theta}$ at the cavity surface

$$
\sigma_{\theta}=\frac{E}{3}\left(\lambda_{a}^{2}-\lambda_{a}^{-4}\right)
$$




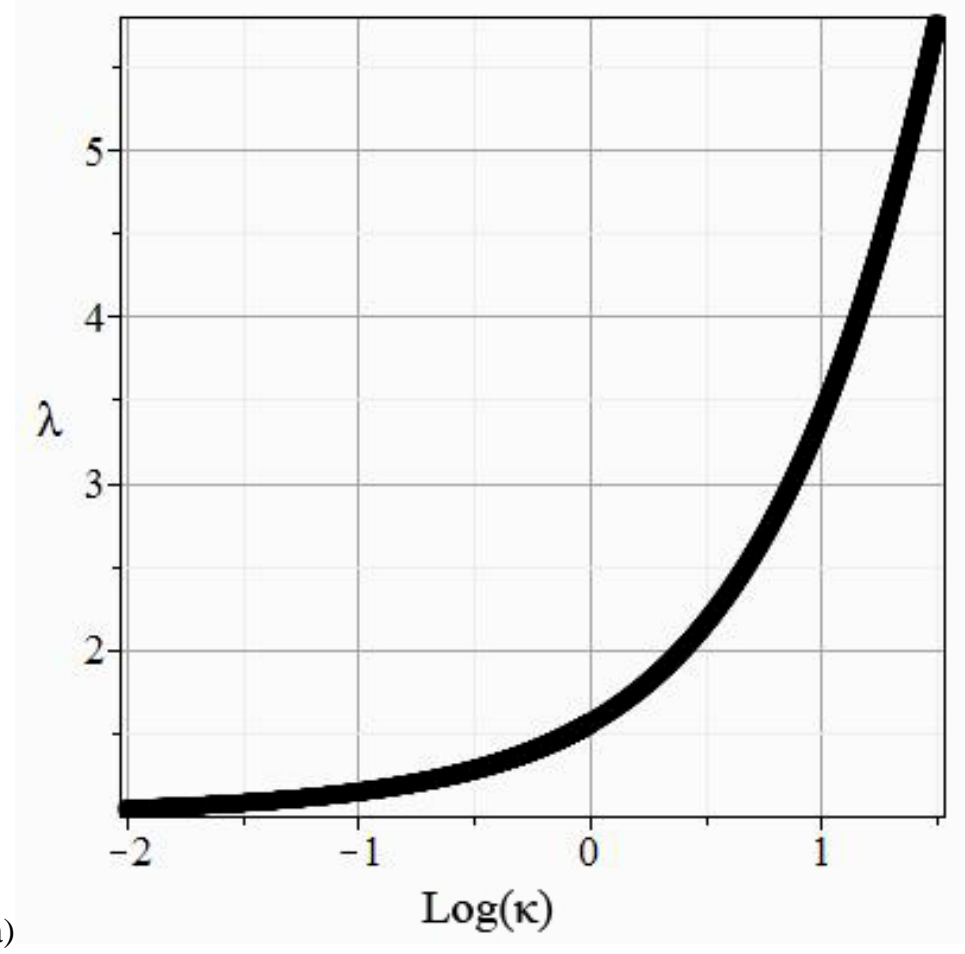

(a)

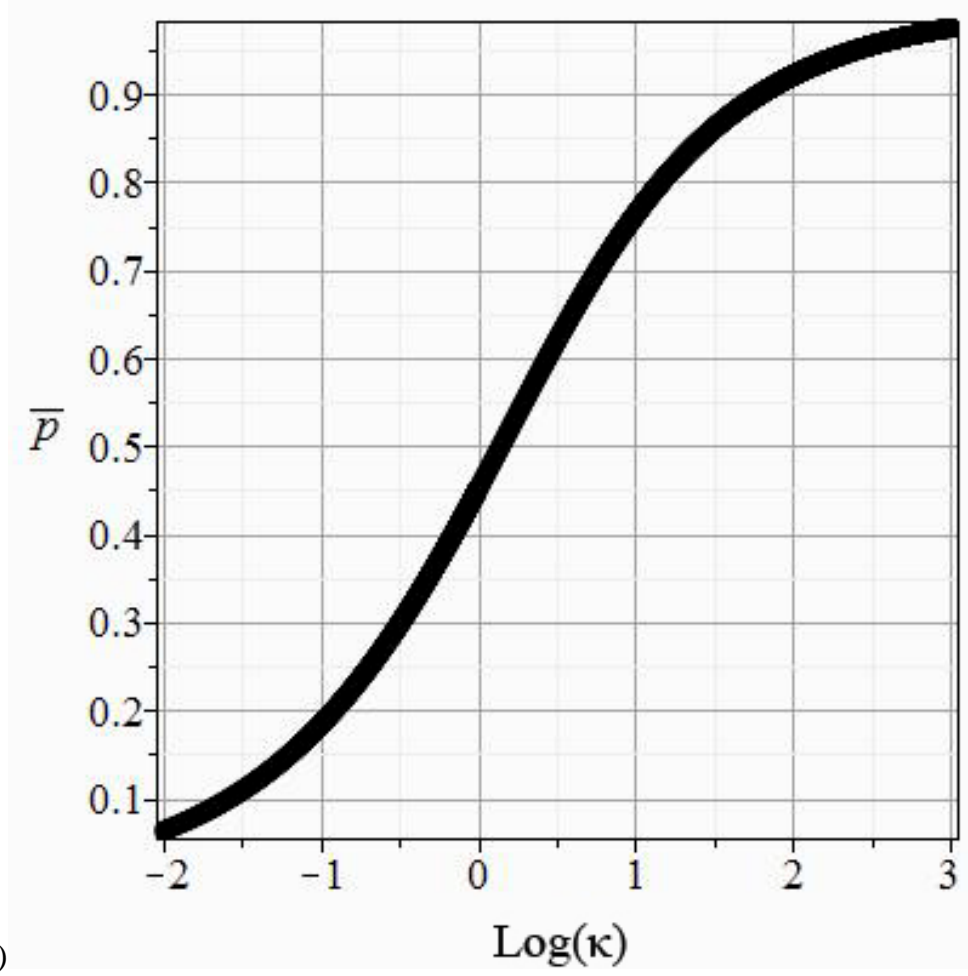

Figure 3: Critical conditions for instability of an initial spherical cavity (a) $\lambda$ vs $\log (\kappa)$, and (b) $\bar{p}=6 p_{c} / 5 E$ ere $\kappa=6 S / E q$.

Since at the condition of failure the critical extension $\lambda_{a}$ is related to the critical pressure $p_{c}$ and the parameter $\kappa$, as shown in Figure 3, equation (44) can be used to relate the circumferential stress at failure to the same quantities. This has been done, using the SCILAB program. For the two limiting values of $\kappa$ the 
circumferential stress can be explicitly related to $p_{c}$ and $\kappa$.

For $\kappa<<1$, and $\lambda_{a c}=1+(\kappa / 6)^{0.5}$ (see eqn (38)) equation (44) yields

$$
\sigma_{\theta}=E \sqrt{\frac{2 \kappa}{3}}=\sqrt{\frac{4 S E}{a_{0}}}
$$

And using equation (29) it follows

$$
\sigma_{\theta}=\frac{3}{2} p_{c}
$$

This last result can easily be verified from classical elastic theory!

\section{RESULTS AND DISCUSSION}

The above analysis provides an equation for determining the critical extension ratio for a given value of a parameter $\kappa$ which defines the ratio of the surface energy and the product of the modulus of elasticity and the radius of the cavity in a incompressible propellant material. From the critical extension ration one can determine the critical radial pressure applied at infinity. The critical flaw sizes were also determined using small and large values of the parameter $\kappa$. It was found that the critical applied pressure is inverse analogous to the square root of the initial size of the cavity for small values of $\kappa$, and is direct analogous to the square root of the initial size of the cavity for large values of $\kappa$. In Biwa's solution (eqn (2)) the initial size of the cavity is not involved in the final relation that describes the critical pressure as a function of the surface energy. The results in Fig. 2 show that for the dead load traction above a certain level, there are multiple equilibrium solutions, the intact configuration and two cavitating configurations. The results in the present paper has been based on the neo- Hookean strain energy function, an extension of this work could be done for other types of strain energy functions e.g. Ogden, Arruda-Boyce etc.

\section{BIBLIOGRAPHY}

[1] BENCHER, C, DAUSKARDT, R, RITCHIE, R., "Microstructural damage and fracture processes in a composite solid rocket propellant”, Journal of Spacecraft and Rockets, v. 32, n.2, pp. 328-334, 1995.

[2] KAKAVAS, P. A., CHANG, W.V., "Acoustic emission in bonded elastomer discs subjected to compression. Part I.”, Journal of Applied Polymer Science, v.45, n.5, pp. 865-869, 1992.

[3] KAKAVAS, P. A., BLATZ, P.J., "Effects of voids on the response of a rubber poker chip sample”, Journal of Applied Polymer Science, v. 43, n.6, pp.1081-1086, 1991.

[4] KINLOCH, A. J., GLEDHILL, R. A., "Propellant failure - A fracture mechanics approach", In: AIAA, SAE, and ASME, Joint Propulsion Conference, 16th, Hartford, Conn.; United States., 30 June-2 July, AIAA 7, 1980.

[5] SCHAPERY, R. A., "Fracture mechanics of solid propellants", In: Fracture mechanics; Proceedings of the Tenth Symposium on Naval Structural Mechanics, Washington, D.C; United States, v.11-13, pp. 387398, 1978.

[6] TRUMEL, H., DRAGON, A., FANGET, A., et al., "A constitutive model for the dynamic and highpressure behavior of a propellant-like material: Part I: Experimental background and general structure of the model”, International Journal for Numerical and Analytical Methods in Geomechanics, v. 25, pp.551-579, 2001.

[7] LIU, C.T., “Crack growth behavior in a solid propellant”, Engineering Fracture Mechanics, v.56, n.1, pp. 127-135, 1997.

[8] HO, S.Y., "High strain-rate impact studies of predamaged rocket propellants. I. Characterization of damage using a cumulative damage failure criterion”, Combustion and Flame, v. 104 ,n.4, pp. 524-534, 1996.

[9] ZENG, JIA-YA., "Study on the Fracture Behavior of HTPB Propellant by Means of SEM", Journal of Solid Rocket Technology, v.22, n.4, 1999.

[10] KUO, K.K., MORECI, J.A., “Crack propagation and branching in burning solid propellants”, In: Sym- 
posium (International) on Combustion, v.21, n.1, pp.1933-1941, 1988.

[11] YU, CHEN., YUN-FEI, LIU, JI-DONG, XIA. "Quantitative experimental study on effect of bonding agents on the dewetting damage of NEPE solid propellant”, Journal of Solid Rocket Technology, v.33, n.3, pp. 299-301, 2010.

[12] XU, F., ARAVAS, N., SOFRONIS, P., "Constitutive modeling of solid propellant materials with evolving microstructural damage", Journal of the Mechanics and Physics of Solids, v. 56, n. 5, pp. 2050-2073, 2008.

[13] GRIFFITH, A. A., "The Phenomena of Rupture and Flow in Solids”, Philosophical Transactions of the Royal Society (London), Series A, v. 221, pp.163-198, 1921.

[14] BALL, J.M., "Discontinuous Equilibrium Solutions and Cavitation in Nonlinear Elasticity”, Philosophical Transactions of the Royal Society (London), Series A, v. 306, pp. 557-611, 1982.

[15] HORGAN, C.O., and POLIGNONE, D.A., "Cavitation in nonlinearly elastic solids: a review", Appl. Mech. Rev., v. 48, pp. 471-485, 1995.

[16] BIWA, S., "Cavitation in finite elasticity with surface energy effects", Int. Journal of Non-Linear Mechanics, v. 41, pp. 1084-1094, 2006.

[17] OGDEN, R.W., Nonlinear elastic deformation, Dover, 1997.

[18] HOLZAPFEL, G.A., Nonlinear Solid Mechanics: A Continuum Approach for Engineering, 1st edition, Wiley, 2000.

[19] GENT, A. N., LINDLEY, P. B., "Internal Rupture of Bonded Rubber Cylinders in Tension”, Proceedings of the Roal Society (London) 1959; series A, v. Z49, pp.195-205.

[20] ABRAMOWITZ, M. and STEGUN, I., Handbook of Mathematical Functions with Formulas, Graphs and Mathematical Tables, National Bureau of Standards, Applied Mathematics Series, section 3.8.2 on p.17, 1964.

[21] GREEN, A. E., ZERNA, W., Theoretical Elasticity, University Press, Oxford. 1992

[22] TIMOSHENKO, S., GOODIER, J. N., Theory of Elasticity, 3rd ed. McGraw-Hill Book Company, New York, 1970. 